\title{
Isolated Spinothalamic Sensory Impairment of the Left Arm following Contralateral Medullary Infarction: A Case Report
}

\author{
Michael Kueper $^{\mathrm{a}} \quad$ Kiriaki Kollia $^{\mathrm{b}}$ Oliver Kastrup $^{\mathrm{a}}$ \\ ${ }^{a}$ Department of Neurology and ${ }^{\mathrm{b}}$ Institute for Diagnostic and Interventional Radiology and Neuroradiology, \\ University of Duisburg-Essen, Essen, Germany
}

\begin{abstract}
Dear Sir,
A 63-year-old smoker noticed a sudden impairment of temperature sensation of the left arm, with coldness associated with a sensation of burning pain. Thermal sensory deficit was also present in the left trunk. The patient did not notice any other accompanying neurological deficit such as diplopia, dysphagia, dizziness or coordination disturbances. Initially the patient was admitted to a cardiology department, where arterial hypertension was diagnosed. Transthoracic echocardiography showed left ventricular hypertrophy with normal ejection fraction (60\%). MRI scan was performed but remained unremarkable (slice thickness $6 \mathrm{~mm}$ ). The patient was referred to our hospital for reevalua-
\end{abstract} tion.

Neurological examination 4 weeks after onset showed decreased thermal sensation, especially of the left arm and, to a lesser degree, of the left trunk. The same regions exhibited moderate hypalgesia. There were no further sensory deficits, including position and vibration sense, discrimination and stereognosis. Examination of the cranial nerves, the motor system and coordination yielded normal results. Cardiovascular risk factors included smoking, hypertension, moderate adiposity and hypercholesterolemia.
Cerebral MRI with thin brainstem slices (thickness $2 \mathrm{~mm}$ ) detected a small ischemic lesion in the right dorsolateral and ventral medulla (fig. 1). MR angiography showed occlusion of the right vertebral artery (fig. 2), as did duplex sonography. Sensory evoked potentials from the median and tibial nerves were not delayed, providing evidence of normal lemniscal sensation.

The patient was discharged with the diagnosis of a lacunar brainstem stroke following occlusion of the right vertebral artery. Medication with aspirin and simvastatin was initiated for the purpose of secondary prevention.

\section{Discussion}

In our patient, impairment restricted to pain and temperature sensation were the only features of a lacunar medullary infarction affecting the spinothalamic tract. There were no other symptoms suggestive of brainstem stroke on examination 4 weeks later. Furthermore, the patient reported no other transient neurological deficit. Nevertheless, it cannot be ruled out that other brainstem symptoms, such as mild Horner's syndrome, were ini- tially present, but not noticed by the patient.

Ischemic stroke with pure sensory symptoms may result from infarction of the brainstem, the thalamus, the lenticulocapsular area/corona radiata or the sensory cortex $[1,2]$. Lemniscal and spinothalamic sensation may be affected together or selectively. In brainstem lacunar stroke, paramedian pontine infarction is the most common cause of pure sensory impairment involving lemniscal sensation [3]. Lateral medullary infarction is a rare cause of isolated contralateral spinothalamic sensory impairment and has been described only twice in the literature $[4,5]$, with the suggestion that it be classified as type IV according to Stopford's classification of sensory loss in lateral medullary infarction $[4,6]$. Most commonly, lateral medullary infarction leads to a sensory deficit according to Wallenberg's syndrome, with loss of temperature and pain sensation in the ipsilateral face and the contralateral hemibody [7].

To our knowledge, this is the first reported case of lateral medullary infarction with isolated pain and temperature impairment following vertebral artery occlusion. Vertebral artery occlusion may be either asymptomatic or may lead to poste-

\section{KARGER}

Fax +4161306 1234

E-Mail karger@karger.ch

www.karger.com (c) 2008 S. Karger AG, Basel

0014-3022/08/0594-0216\$24.50/0

Accessible online at:

www.karger.com/ene
Michael Kueper

Department of Neurology, University of Duisburg-Essen

Hufelandstrasse 55, DE-45147 Essen (Germany)

Tel. +49201723 2584, Fax +492017232127

E-Mail Michael.Kueper@uni-duisburg-essen.de 
Fig. 1. Thin-slice axial (a) and sagittal (b) $\mathrm{T}_{2}$ images show a right dorsolateral medullary infarct in the retro-olivary medullary tegmentum.

Fig. 2. MR time-of-flight angiography 3dimensional reconstruction (a) and MR angiography (b) show occlusion of the distal right vertebral artery.

Fig. 3. Enlarged view of the infarct and corresponding anatomical drawing. LST = Lateral spinothalamic tract.

rior circulation stroke or transient ischemic attack [8]. Occlusive disease of the vertebral artery represents the most common cause of lateral medullary infarction $[7,9$ 11]. In this atypical presentation, an active
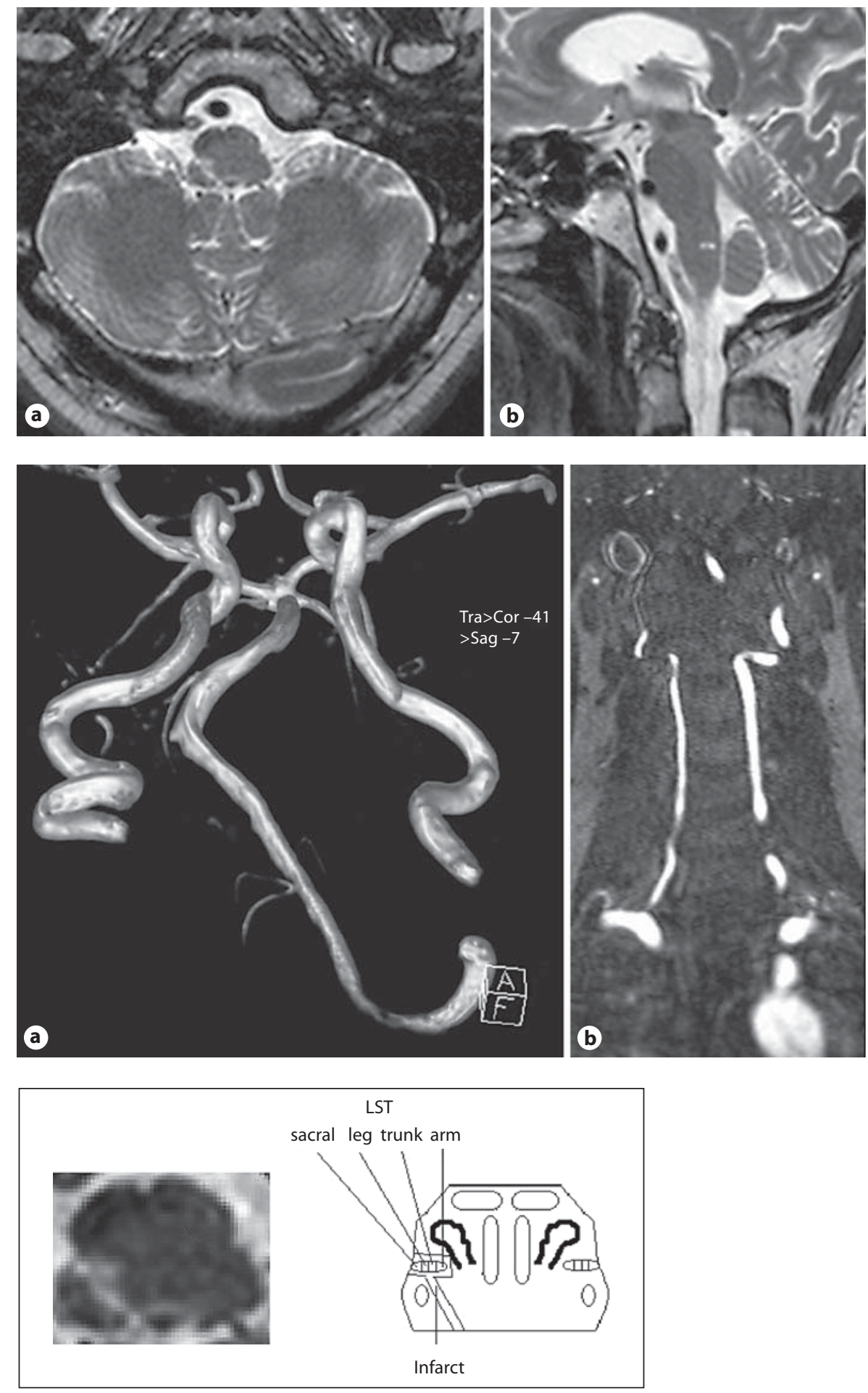

search for a cardiac or arterial cause of the stroke is indicated. In our patient, occlusion of the distal right vertebral artery suggestive of atherosclerotic-thrombotic occlusion was found using MR angiography and duplex ultrasound examination. Atheromatous intima thickening was seen in both internal carotid arteries in duplex sonography, without significant stenosis. $\mathrm{Pa}$ tient cardiovascular risk factors included 
smoking, adiposity, hypercholesterolemia, and hypertension. Aside from atheroma, cardioembolism and dissection are frequent causes of vertebral artery occlusion [7] in patients with medullary infarction. Transthoracic echocardiography showed left ventricular hypertrophy with normal ejection fraction, but did not point to cardioembolic stroke etiology. ECG did not suggest atrial fibrillation.

Thin-slice $(2 \mathrm{~mm})$ MRI scan showed dorsolateral infarction of the right middle medulla oblongata. The lesion was visible on one sagittal and two axial slices only. Thus, it is not surprising that the infarct was not evident on the MRI scan (slice thickness $6 \mathrm{~mm}$ ) performed 4 weeks before admission to our hospital. In lacunar brainstem lesions, thin-slice MRI is likely to demonstrate higher sensitivity and has been suggested in standard imaging protocols for brainstem ischemia [12].

The patient had contralateral spinothalamic sensory deficit, including the arm and trunk. Cerrato et al. [13] reported spinothalamic sensory impairment of the left arm and neck combined with lemniscal sensory impairment of the right side in a patient with infarction of the lower lateral medulla. The authors concluded that a lesion was present in the right medial fibers of the spinothalamic tract representing the arm and neck. In our patient, the lesion extended to more superficial spinothalamic fibers, including the representation of the left trunk. Figure 3 shows an enlarged view of the infarction in relation to an anatomical drawing of the medulla oblongata at this level. The lesion correlates well with the location of the lateral spinothalamic tract. Although clinically not affected in our patient, involvement of superficial fibers from the sacral region and the leg could be expected from the le- sion's location. However, it has been reported that sensory findings in brainstem lesions do not always correspond well with their anatomical site $[14,15]$.

Arterial blood is supplied to the medulla by perforating branches arising from the vertebral artery, anterior and posterior spinal artery, anterior inferior and posterior inferior cerebellar artery and the basilar artery [16]. Those perforators are divided into distinct subgroups (anteromedial, anterolateral, lateral and posterior). Infarct location in our patient suggests the involvement of branches of the lateral group arising from the vertebral or posterior inferior cerebellar artery.

In conclusion, our report shows the diversity of pure sensory stroke in the brainstem. Although lateral medullary infarction is rarely connected with restricted spinothalamic impairment, it should be considered an infrequent cause of pure sensory stroke.

\section{References}

1 Kim JS, Lee MC: Stroke and restricted sensory syndromes. Neuroradiology 1994;36: 258-263.

2 Kim JS: Pure sensory stroke. Clinical-radiological correlates of 21 cases. Stroke 1992;23: 983-987.

3 Kim JS, Bae YH: Pure or predominant sensory stroke due to brain stem lesion. Stroke 1997;28:1761-1764.

4 Vaudens P, Bogousslavsky J: Face-armtrunk-leg sensory loss limited to the contralateral side in lateral medullary infarction: a new variant. J Neurol Neurosurg Psychiatry 1998;65:255-257.

5 Blitshteyn S, Rubino FA: Pure sensory stroke as an isolated manifestation of the lateral medullary infarction. J Neuroimaging 2005; 15:82-84
6 Stopford JSB: Arteries of pons and medulla oblongata. J Anat 1924;59:120-128.

7 Vuilleumier P, Bogousslavsky J, Regli F: Infarction of the lower brainstem: clinical, aetiological and MRI-topographical correlations. Brain 1995; 118:1013-1025.

8 Hennerici M, Aulich A, Sandmann W, Freund H-J: Incidence of asymptomatic extracranial artery disease. Stroke 1981;12: 750-758.

9 Wityk RJ, Chang HM, Rosengart A, Han WC, DeWitt D, Pessin MS, Caplan LR: Proximal extracranial vertebral artery disease in the New England Medical Center Posterior Circulation Registry. Arch Neurol 1998;55: 470-478.

10 Kim JS, Lee JH, Choi CG: Patterns of lateral medullary infarction. Vascular lesion-magnetic resonance imaging correlation of 34 cases. Stroke 1998;29:645-652.

11 Fisher CM, Karnes WE, Kubik CS: Lateral medullary infarction: the pattern of vascular occlusion. J Neuropathol Exp Neurol 1961; 20:323-379.

12 Schulte-Altedornenburg G, Brückmann $\mathrm{H}$ : Imaging techniques in diagnosis of brainstem infarction. Nervenarzt 2006;77:731744.

13 Cerrato P, Imperiale D, Bergui M, Giraudo M, Baima C, Grasso M, Lopiano L, Bergamasco B: Restricted dissociated sensory loss in a patient with a lateral medullary syndrome. Stroke 2000;31:3064-3066.

14 Cerrato P, Baima C, Bergui M, Grasso M, Lentini A, Giraudo M, Azzaro C, Bergamasco B: Restricted pain and thermal sensory loss in a patient with pontine lacunar infarction: a clinical MRI study. Eur J Neurol 2005; 12:564-565.

15 Helgason CM, Wilbur AC: Basilar branch pontine infarction with prominent sensory signs. Stroke 1991;22:1129-1136.

16 Tatu L, Moulin T, Bogousslavsky J, Duvernoy $\mathrm{H}$ : Arterial territories of human brain: brainstem and cerebellum. Neurology 1996; 47:1125-1135. 